\title{
Nuevas perspectivas de la diálisis peritoneal: ultrafiltración a un paciente con insuficiencia cardiaca refractaria a diuréticos
}

\author{
Francisca Gruart Armangué, Esther Salillas Adot, Neus Simal Vélez, Maricel Julve Ibáñez, Alex Andujar Asensio, \\ $M^{a}$ José Argerich González
}

Unidad de Diálisis Peritoneal. Servicio de Nefrología. Hospital Universitari de Bellvitge-IDIBELL. Universitat de Barcelona

\section{Introducción}

Una de las particularidades que desarrolla el síndrome cardiorenal (SCR) es un círculo en el que como consecuencia del bajo gasto cardíaco hay alteración en el equilibrio del óxido nítrico y en la liberación de la hormona antidiurética, entre otros; la inducción a la vasoconstricción renal y el aumento en la reabsorción en el túbulo proximal de agua y sodio reduce el aporte de agua y sodio al nivel del túbulo distal, lo que provoca la disminución en la diuresis del péptido natriurético y aumenta la sensibilidad a la acción de la aldosterona (secretada por las glándulas suprarrenales) que actúa en la conservación del sodio y secretando potasio e incrementando la presión sanguínea). Esto explica que en los pacientes con insuficiencia cardíaca (IC), una de las características más evidentes es la hipervolemia y la retención de sodio.

Datos recientes apoyan que, en el $\mathrm{SCR}$, la retención de líquidos, de sodio y la posterior resistencia a los diuréticos, provocan una sobrecarga progresiva del volumen y empeoramiento de la IC, con reducción de gasto cardíaco y mayor disfunción renal. En consecuencia, se genera una situación con mal pronóstico y pocas opciones alternativas ${ }^{1}$.

La diálisis peritoneal, usada en la insuficiencia renal crónica (IRC), tiene por objetivo la eliminación de productos de desecho y agua, mediante la difusión y la convección. La eliminación de sodio es mayor

\section{Correspondencia: \\ Francisca Gruart Armangué \\ Servicio de Nefrología \\ Hosp. U. de Bellvitge \\ C/. Feixa Llarga s/n. 08907 \\ Hospitalet de Llobregat. Barcelona \\ E-mail: pgruart@serveisnet.com}

con soluciones de dextrosa hipertónica durante el tiempo de permanencia intraperitoneal pero esta propiedad se pierde durante un período largo. Además la hipertonicidad del líquido produce mayor daño a la membrana peritoneal. La aparición de la solución con icodextrina, polímero de glucosa de elevado peso molecular que es isoosmolar, ha favorecido por su efecto oncótico la producción de mayor ultrafiltración, reabsorbiéndose más lentamente que los líquidos con dextrosa permitiendo, así una mayor permanencia intraperitoneal (de 10 a 14 horas) y consecuentemente una mayor eliminación del sodio.

Estos nuevos tratamientos que están surgiendo permiten mejorar la calidad de vida, la situación clínica y la supervivencia de los pacientes que han desarrollado resistencia a los diuréticos. Pueden ser beneficiosos en situaciones en que el SCR es agudo, pero no parece tener demasiado éxito cuando la insuficiencia cardíaca congestiva (ICC) es crónica.

La New York Heart Association² (NYHA), define cuatro clases funcionales de IC según sea la actividad física del paciente y la severidad de los síntomas. (Tabla I)

Tabla I. Clasificación de la CI según la NYHA (New York Heart Association)

\begin{tabular}{c|l}
\hline $\begin{array}{c}\text { Clase } \\
\text { I }\end{array}$ & $\begin{array}{l}\text { No limitación de la actividad física. La actividad ordinaria no } \\
\text { ocasiona excesiva fatiga, palpitaciones, disnea o dolor anginoso. }\end{array}$ \\
\hline $\begin{array}{c}\text { Clase } \\
\text { II }\end{array}$ & $\begin{array}{l}\text { Ligera limitación de la actividad física. Confortables en re- } \\
\text { poso La actividad ordinaria ocasiona fatiga, palpitaciones, } \\
\text { disnea o dolor anginoso. }\end{array}$ \\
\hline $\begin{array}{c}\text { Clase } \\
\text { III }\end{array}$ & $\begin{array}{l}\text { Marcada limitación de la actividad física. Confortables en } \\
\text { reposo. Actividad física menor que la ordinaria ocasiona fa- } \\
\text { tiga, palpitaciones, disnea o dolor anginoso. }\end{array}$ \\
\hline Clase \\
IV & $\begin{array}{l}\text { Incapacidad para llevar a cabo cualquier actividad física sin } \\
\text { molestias. Los síntomas de insuficiencia cardíaca o de sín- } \\
\text { drome anginoso pueden estar presentes incluso en reposo. } \\
\text { Si se realiza cualquier actividad física, el malestar aumenta }\end{array}$ \\
\hline Comité de criterios NYHA, 1964.
\end{tabular}

Las técnicas de ultrafiltración extracorpórea y la diálisis peritoneal demuestran: 
- Mejoría de la clase funcional ${ }^{3-4}$ del paciente.

- Reducen los ingresos hospitalarios.

- Mejoran la percepción de calidad de vida.

- Disminuye la mortalidad.

- Reducción del coste en tratamiento5.

\section{Historia clínica}

Presentamos el seguimiento de un paciente de 52 años, con diabetes mellitus (DM) tipo I desde los 28 años, con irregular control metabólico; con hipercolesterinemia familiar y claudicación intermitente y exfumador. Debuta con cardiopatía isquémica a los 33 años. Este seguimiento es descriptivo longitudinal en un tiempo entre 6 meses pre y post al inicio del tratamiento con diálisis peritoneal intermitente nocturna (IPDN), con un único recambio peritoneal de icodextrina y una permanencia intraperitoneal de unas $12 \mathrm{~h}$.

10/01/2012: Ingreso hospitalario de 7 días por disnea progresiva, edemas en extremidades inferiores $y$ múltiples episodios de ortopnea y disnea paroxística nocturna. Clasificación NYHA: II - III. Se optimiza tratamiento diurético. Alta hospitalaria.

28/01/2012: Ingreso hospitalario de 11 días. Presenta palpitaciones tras esfuerzo físico con mareos sin pérdida de conciencia y dolor centrotorácico opresivo de gran intensidad que irradia ambos brazos y hombros. IC biventricular. Clasificación NYHA III-IV. Tras exploraciones pertinentes, se plantea la posibilidad de implantación de un desfibrilador automático implantable (DAI), a valorar de forma ambulatoria. Se optimiza tratamiento diurético y alta hospitalaria.

23/03/2012: Ingresode 12 días. Presentaempeoramiento de la disnea con edemas maleolares y ganancia de $6 \mathrm{Kgr}$ de peso. Expectoración hemoptoica y marrón (doble tratamiento anticoagulante y antiagregante) e hipertensión arterial pulmonar (HTAP). Tratamiento de la HTAP y antibioterapia.

Se descarta trasplante cardiaco por DM tipo I.

Seguirá revisiones ambulatorias.

10/06/2012: Ingreso hospitalario de 41 por aumento de edemas en extremidades inferiores y escrotal. Aumento de peso de $10 \mathrm{Kg}$. Disnea progresiva hasta en reposo con ortopnea. Presenta descenso de la función renal (Urea:
$16 \mathrm{mml} / \mathrm{L} ;$ Cr: $138 \mu \mathrm{m} / \mathrm{L})$. Clasificación NHYA IV. Se implanta el DAI tricameral. Se administran diuréticos ev a altas dosis, produciéndose un deterioro funcional renal con disminución de la diuresis.

Se implanta catéter peritoneal Tenckhoff recto dos cuff, por incisión percutánea. (23/07/2012).

15/08/2012: Inicio de la IPDN con icodextrina $1^{\prime} 5 \mathrm{~L}$ con permanencia IP de $11 \mathrm{~h}$. A los siete días empieza a ponerse los $2 \mathrm{~L}$ IP y misma permanencia.

Los días totales de ingreso han sido de 71 días durante estos seis meses descritos. Diuresis $\pm 2500 \mathrm{ml}$; peso 78 $88 \mathrm{Kg}$; clase funcional habitual NYHA II-III/IV.

\section{Resultados}

Los datos desde los 6 meses del inicio de la DP, son:

- Días de ingreso: 0 .

- Diuresis $\pm 2500 \mathrm{ml} /$ día; Función renal mantenida. Urea: $\pm 9,77 \mathrm{mmol} / \mathrm{L} ; \mathrm{Cr}: \pm 109,5 \mu \mathrm{mol} / \mathrm{L} ;$ UF: \pm $500 \mathrm{ml} / \mathrm{día}$.

- Peso: $72,5 \mathrm{Kg}-78,5 \mathrm{Kg}$; Edemas en EEII $(+)-(++)$

- TA: $\pm 120 / 70$.

- Al inicio de la DP se consiguió rebajar los diuréticos a $3 \mathrm{cp} / d i ́ a$ (llegó a tomar $7 \mathrm{cp} / d i ́ a)$, siendo la dosis actual $4 \mathrm{cp} / d i ́ a$.

- A los dos meses del inicio de DP sufre una peritonitis por germen de origen intestinal (bacteroides thetaiotaomicron) coincidiendo con disminución del ritmo deposicional que curó con tratamiento antibiótico.

- El paciente verbaliza su mejoría en calidad de vida a la cual había renunciado: realiza una vida activa sin disnea y ha mejorado su apetito y su vida social.

\section{Conclusión}

Queda demostrado que:

- No hay ingresos hospitalarios.

- Mejora la calidad de vida, percibida por el paciente.

- Hay aumento del apetito.

- Se conserva la función renal dentro de la normalidad.

\section{Nuestro futuro objetivo es:}

Pensamos que es importante ampliar la experiencia para poder afirmar que la DPI puede ser una buena alternativa para el tratamiento de la ICC refractaria. 
Recibido: 10 Febrero 2014

Revisado: 10 Febrero 2014

Modificado: 10 Febrero 2014

Aceptado: 10 Febrero 2014

\section{Bibliografía}

1. Nuñez, J. González, M. Miñana, G. Y cols. Diálisis peritoneal ambulatoria continua y evolución clínica de pacientes con insuficiencia cardíaca congestiva refractaria. Rev Esp Cardiolog. 2012; 65(11): 986-995.

2. Criteria Committee, New York Heart Association. Diseases of the heart and blood vessels. Nomenclature and criteria for diagnosis, 6th ed.Boston: Little, Brown and co, 1964;114.

3. Comité multidisciplinar de expertos. Tratamiento de la IC refractaria con diálisis peritoneal. Recomendaciones basadas en la evidencia científica y experiencia clínica. Fundación Gaspar Casal. Madrid, 2011.

4. Ronco C, Bellomo R, McCullough PA (eds): Cardiorenal Syndromes: An Executive Summary from the Consensus Conference of the Acute Dialysis Quality Initiative (ADQI). EN: Ronco,C. Bellomo, R. McCullough P.A. Cardiorenal Syndromes in Critical Care. Ed. Basel, Karger, 2010, vol 165, pp 54-67.

5. Sanchez,E. Rodríguez,C. Ortega, T. Diaz-Molina,B. Garcia-Cueto,C. Tratamiento de la sobrecarga de volumen en la insuficiencia cardíaca refractaria. Insuficiencia Cardiaca, 2011. Vol 6(1): 19 - 26.

6. Jentzer, Jacob C. DeWald, Tracy A. Hernández, Adrian F. Combination of loop diuretics with thiazide. Type diuretics in heart failure. Journal American College of Cardiology (JACC). 2010, 56: 1527-1534. 